\title{
Lean and Computerized Management System for Non- Hospital-Owned Medical Equipment in Hospital
}

\author{
By Y. Y. Wu, K. Zheng, B. P. LI, C. X. Zheng
}

Department of Purchasing/Logistical/Clinical Engineering, the Children's Hospital Zhejiang University School of Medicine/ National Clinical Research center for child health, Hangzhou 310052, China

\begin{abstract}
Many challenges exist in the management of non-hospital-owned medical equipment. This paper proposes implementing a novel kind of lean and computerized management method, including the management policy, procedures, agreement signing, equipment installation, acceptance and maintenance, and exit procedure. The result shows that the Lean and computerized management system can improve oversight and assure the safe integration of non-hospital-owned equipment to reduce liability exposure and increase compliance with regulations.

Keywords - Non-Hospital-Owned Medical Equipment, Lean Management, computerized management system, Trial Protocol, Medical Safety, assets control.

Copyright (C) 2021. This is an open-access article distributed under the terms of the Creative Commons Attribution License (CC BY): Creative Commons - Attribution 4.0 International - CC BY 4.0. The use, distribution or reproduction in other forums is permitted, provided the original author(s) and the copyright owner(s) are credited and that the original publication in this journal is cited, in accordance with accepted academic practice. No use, distribution or reproduction is permitted which does not comply with these terms.
\end{abstract}

\section{INTRODUCTION}

With the rapid development of the modern hospital, increasing demand for the medical equipment and technology experienced both in clinical and research environments. Due to the diversity of novel medical equipment, some are supplied for free by the vendors. Vendors have a variety of reasons for bringing in their equipment. They may bring in as a service loaner or as a new model to demonstrate to clinicians. As this equipment may be left for weeks for a clinical trial and evaluation only, the new products' technical parameters and performance can be evaluated and compared in hospitals ${ }^{1}$. We define this kind of equipment as trial equipment in this article. The trial equipment we refer to here is not medical equipment in clinical trials for pre-market approval but equipment already approved for the market. All the indirect hospital purchased equipment are treated as non-hospital owned equipment, including trial equipment, research collaboration equipment, household appliances, among other things.

The state of non-hospital-owned equipment is difficult to judge at times, and some of them may not meet the safety requirements, which can lead to safety problems ${ }^{2}$. Valid concerns about non-hospital owned medical equipment include safety and effectiveness, compliance with applicable accreditation standards and legal requirements, proper integration, and technical support. Therefore, they should be under the oversight of clinical engineering, and 
all medical equipment should receive any needed attention regardless of their ownership. In our hospital, all non-hospital-owned equipment is supervised by a Lean and computerized management system. This article takes the trial equipment we defined before as an example and describes the relevant management program.

\section{MATERIALS}

Lean management is designed to enhance productivity by improving work efficiency ${ }^{3}$. During the whole procedure, the manager's duty is specified and explicit, and the management process is standardized and systematized. On the one hand, the Lean management's core idea is applied in non-hospital-owned medical equipment, and a strict management policy and procedure ensure its safety. On the other hand, information technology is applied to develop a customized tool for implementing the overall non-hospital owned medical equipment management ${ }^{4}$. Our hospital's medical equipment management system includes the management functions of hospital-wide medical equipment such as testing, reporting for repair, and maintenance, among which the management of nonhospital owned medical equipment is an important module.

\section{A. Design a Rigorous Management Policy and Procedure}

Before its first clinical use, all non-hospital-owned medical equipment providers need to sign an agreement or protocol with the hospital represented by the clinical engineering department. Any unauthorized or disapproval use of non-hospital-owned medical equipment is strictly forbidden. Hence, the recommended procedure is shown in Figure 1.

Step 1: Trial application. The corresponding department is asked to complete the medical device clinical trials' application form, with a description of application reasons, device name, brand and model, quantity, regulation registration certificate number, vendor name.

Step 2: Legal compliance examination and verification. All the necessary documents are reviewed by the department of clinical engineering, including the medical device registration certificate issued by the China Food and Drug Administration (CFDA), production enterprise license, business certificate business license, factory authorization letter, operation manuals. The copies of these documents are saved for future reference.

Step 3: Approving or comments by the related functional departments such as the medical administration department and finance department.

Step 4: Submission to hospital medical equipment management committee for consideration.

Step 5: Signing a protocol of authorized usage for the non-hospital owned medical equipment.

Step 6: Labeling the device and informed users.

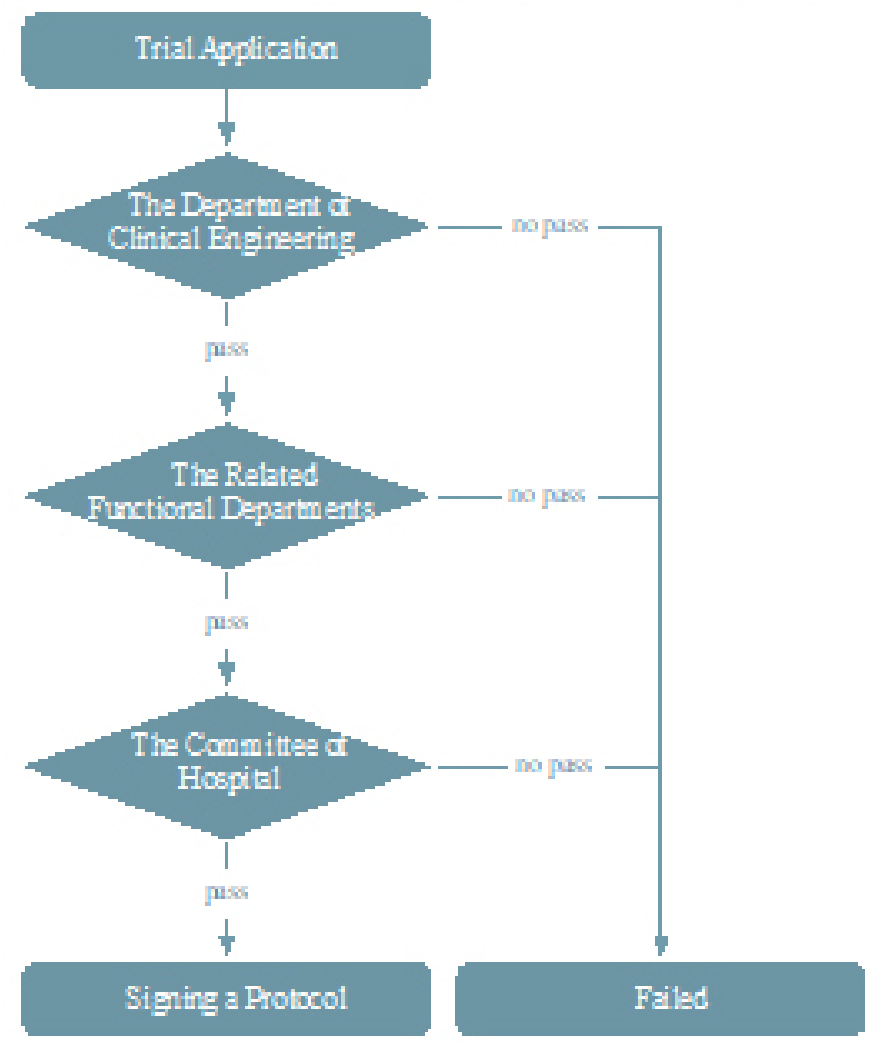

FIGURE 1. The access protocol for non-hospital owned medical equipment.

\section{B. Sign Medical Equipment Trial Protocol}

The trial protocol is signed by the hospital (Party A) and the vendors such as the sales company or manufacturer (Party B), in which the duties and obligations for both sides and the agreed usage duration (usually no 
more than 3 months) are specified. According to the trial protocol, Party B's equipment should be conforming to all the regulations made by CFDA. Also, Party B should provide valid certificates, installation procedures, and user training. When the trial period ends up, the equipment should return to Party B in time. Party A should take good care of the devices during the trial period. If the device breaks due to the user's carelessness or abuse, the hospital (Party A) will be responsible for the compensation.

\section{Standardization of Installation and Acceptance Process}

When the medical equipment trial protocol is implemented, the supplier shall be responsible for the trial equipment installation, and the engineers of the department of clinical engineering at the hospital will make an acceptance check to ensure the safety of the equipment.

After the completion of the acceptance process, the supplier needs to conduct training for potential users. Some of the non-hospital-owned equipment is surgical instruments in the operating room. Such instruments may be used already many times in different hospitals. Therefore, it might cause potential surgical infection if sterilization protocol is not carried out strictly or adequately ${ }^{5}$. For example, in orthopedic implant surgery, if the bacteria contaminate the implant, it will be quite challenging to tackle this problem since a layer of protective film is generated in the implant surface, which makes the antibiotics useless and brings about great pain for the patients ${ }^{6}$. Hence, additional precautions should be taken, and relevant measures should be taken according to hospital infection control policy if the trial device is the surgical instrument ${ }^{7,8}$. The corresponding training, assembly, and disassembly demonstration should be provided for the central sterile supply department staff to ensure that all the operations conform to the disinfection and sterilization requirements ${ }^{9,10}$ and make sure that the instruments are used in a safe situation ${ }^{11}$.

\section{The Application of Non-Hospital Owned Medical Equipment Management Module}

After completion of the acceptance and training procedures, the trial equipment is commissioned. Simultaneously, all the useful information will be loaded into the computerized management system by the clinical engineering department staff, including basic info, clinical department, maintenance record, and the trial's validity period. Besides, a QR code label containing affiliation, equipment name, brand and model, serial number, and the clinical department is labeled on each piece of trial equipment (Figure 2).

\section{Children's Hospital of Zhejiang}

University School of Medicine

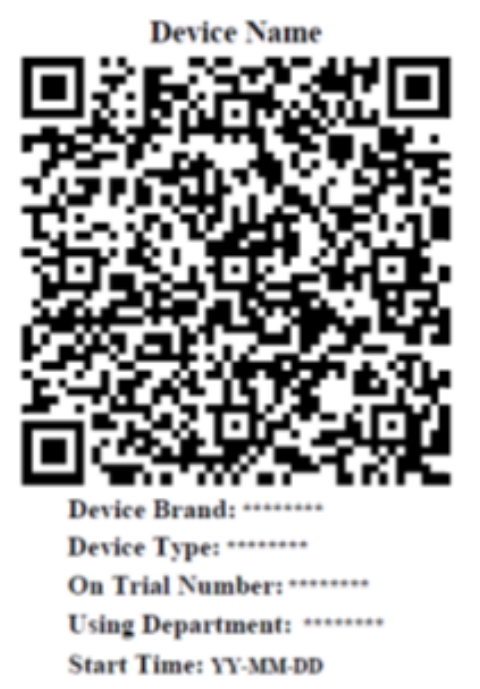

FIGURE 2. The QR code label.

In this paper, a lean and computerized management system is proposed and implemented in the hospital, in which non-hospital owned equipment is subject to oversight and control in a standardized framework, especially in terms of the following several aspects.

1. Based on the strict management policy and procedure, some unnecessary and less prominent medical devices are filtered in the approval process. The quantity of external medical equipment is better controlled and quality is better guaranteed. There are no more than 10 cases of trial medical equipment in our hospital every year in recent years.

2. QR code label. More detailed information is obtained following the scanning of the $Q R$ code. It contains device type, serial number, registration certificate information. Besides, repair and maintenance records can be documented in the computerized management system. 
The registration certificate period for non-hospitalowned equipment will be monitored by the clinical engineering department's computerized management system. If the clinical department applies for extending the trial time, its validity period must be reviewed and confirmed. Hence, the working efficiency is enhanced since the validity of the registration certificate and trial period validity can be checked by scanning the QR code.

3. Planned maintenance. The computerized management system alerts the need for any maintenance due three days in advance, at which time the department of clinical engineering will contact the vendors for a timely inspection and maintenance. The maintenance task and its record can be easily accessed and carried out by scanning the QR code. All the corresponding information can be reviewed by logging in the system.

4. End of the trial. The computerized management system reminds the trial ending time three days in advance. The clinical engineering department is responsible for reminding and contacting the vendors to remove the trial equipment from the hospital. Also, it is recorded in the information system.

\section{CONCLUSIONS}

The lean management system we described takes key management elements of non-hospital owned medical equipment into consideration. Combined with the government requirements of rules and regulations with hospital real-practice scenarios, the department of clinical engineering has designed and implemented an effective lean management system for non-hospital-owned medical equipment. Furthermore, the whole management process is carried out with the support of an information system, in which all the corresponding information and certificates, and quality control activities are recorded and is reviewed together conveniently. By doing so, the management efficiency and performance improves. The risks and potential damages from non-hospital-owned medical equipment are effectively mitigated; medical safety for hospitals and patients is enhanced; compliance with regulations increases.

\section{ACKNOWLEDGMENT}

This work was supported by National Key Research and Development Project (2017YFC0114107). We want to extend our sincere gratitude to Dr. Yadin David for his revising this paper. We are also deeply indebted to our other clinical engineering staff that supported our work.

\section{CONFLICT OF INTEREST}

The authors declare that they have no conflict of interest.

\section{REFERENCES}

1. Shi W J, Kang D H, Tong Q R et al. (2015) Risk Control for the Use of Introduced Trial Medical Equipment. China Medical Devices ISTIC 30(1):125-126.

2. Liu J Y, Li N, Cai M et al. (2017) Evaluation of preventive effect of the management of foreign medical instruments on surgical site infections in orthopedic surgery. Chinese Journal of Nosocomiology 027(012):2849-2852.

3. Collar R M, Shuman A G, FEINER S, et al. (2012) Lean Management in Academic Surgery. Journal of the American College of Surgeons 214(6): 928-936.

4. Huang W (2018) Review of the Development Status of Domestic Hospital Information System. Computer Knowledge and Technology 014(021):89-90,109.

5. Qian W J, Qian J J, Wang W (2018) Investigation and Analysis on the management of surgical instruments in hospital. Shanghai Nursing 104(08):69-72.

6. Epstein A K, Pokroy B, Seminara A, et al. (2011) Bacterial Biofilm Shows Persistent Resistance to Liquid Wetting and Gas Penetration. Proceedings of the National Academy of Sciences 108(3): 995-1000.

7. Wang Z H (2018) The effect of quality traceability management system for disinfection supply on the whole process monitoring of external medical devices. China Medicine and Pharmacy 8(19):183-185+250.

8. Gao Y H, Chen Y W, Wang Y L (2018) Sterilization time of loaner instrumentation by structure and materials. Academic Journal of Chinese PLA Medical School 039(003): 218-222. 
9. Ministry of Health of the People's Republic of China (2009) The Central Sterile Supply Department in Hospital, Part 2. China Standards Press.

10.Organization W H (2014) Practical guidelines for infection control in health care facilities. Manila: WHO Regional Office for the Western Pacific.
11.Li S Y, He X Y, Huang G J (2015) Cleaning and Management of External Surgical Instruments in CSSD. For All Health 9(9):279-280. 